\title{
Pruebas diagnósticas en alergia y su utilidad clínica.
}

\author{
Diagnostic Tests and their Clinical Use in Allergy.
}

\author{
ARRUDA CHAVES Erika*
}

\section{SUMMARY}

The prevalence of allergic diseases in Peru as well as in the rest of the world is increasing every year, independently of any integral treatment and control measures instituted in the last few years. Consequently, direct and indirect costs in the public or private health systems are highly expensive and generate a global concern related with the search for prevention and early diagnosis, which will permit specific actions that minimize disabling states due to this reason. A detailed clinical history is the key to the proper identification and/or dismissal of the probable allergenic agents that will trigger many of the allergic states, as well as indicating the auxiliary diagnostic tests to be chosen. For the different allergic diseases, various methods exist that can be used to help in diagnosis with proven specificity and sensibility, considering the different allergenic agents. Precise identification of such agents permits the introduction of secondary and tertiary prevention methods, which have as primordial purpose to avoid symptoms and exacerbations, preventing disabling states and improving patient life quality. (Rev Med Hered 2004; 15:113-117).

KEYWORDS: Allergy, diagnostic proves, alergens.

\section{INTRODUCCIÓN}

La prevalencia de las enfermedades alérgicas en todo el mundo, incluyendo el Perú, aumenta a cada año, a pesar de las medidas de control y tratamiento integral instituídas en los últimos años. En algunos países, uno de cada cuatro individuos tiene asma u otra enfermedad alérgica. Los costos directos e indirectos del tratamiento de enfermedades alérgicas tanto para el sistema de salud privado como para el sistema público son muy altos y generan una preocupación global hacia la búsqueda de métodos de diagnóstico precoz y de prevención, que permitan acciones que minimicen la generación de discapacidades (1).

Tecnológicamente se ha progresado de forma considerable en el desarrollo de nuevos métodos diagnósticos de las enfermedades alérgicas. No obstante la historia clínica es fundamental en la sospecha de estas enfermedades, la identificación de los alérgenos no es posible sino a través de las pruebas diagnósticas auxiliares.
Para enfermedades como el asma, la rinitis alérgica, la conjuntivitis alérgica, la dermatitis atópica, la dermatitis de contacto alérgica, la urticaria y las alergias alimentarias, hay varios métodos que pueden ser empleados con fines diagnósticos. Son métodos con especificidad y sensibilidad reconocidas; la seguridad de éstos está directamente relacionada con la habilidad técnica profesional, la calidad del material utilizado, el que debe ser estandarizado, y la correcta interpretación de los resultados. Algunos medicamentos como los antihistamínicos y corticoides orales deben ser evitados antes de la realización de las pruebas, para no falsear los resultados. Los objetivos básicos de la realización de las pruebas diagnósticas en enfermedades alérgicas están resumidas en la tabla $\mathrm{N}^{\circ} 1$. De forma general estas pruebas son importantes para determinar o descartar los desencadenantes alergénicos del cuadro clínico en investigación.

Además de su finalidad diagnóstica, las pruebas en alergia también tienen como objetivo la indicación y el 
Tabla $\mathbf{N}^{\circ}$ 1. Finalidades de las pruebas diagnósticas en alergia.

- Determinar el alérgeno relevante estableciendo la causa específica de los síntomas y el grado de sensibilización del individuo.

- Descubrir alergias previamente no sospechadas.

- Diagnóstico diferencial con enfermedades no alérgicas.

- Direccionar el tratamiento para reducir o evitar exposición e indicar desensibilización.

control de tratamiento específico. En casos donde se identifica el factor alergénico relevante desencadenante de la sintomatología del paciente, se puede indicar el adecuado control ambiental, para evitar exposiciones a los alérgenos y por lo tanto, evitar exacerbaciones de la sintomatología. La inmunoterapia específica es otro método terapéutico que puede ser instituído en casos particulares, cuando se identifica el desencadenante alergénico a través de estas pruebas. El estudio control para evaluar la disminución de sensibilización con el tratamiento específico también se realiza utilizándose las pruebas diagnósticas en alergia.

En este artículo serán discutidas las principales pruebas diagnósticas disponibles en nuestro medio con sus respectivas indicaciones, basándose en la especificidad, sensibilidad y seguridad de cada método.

\section{PRUEBAS DIAGNÓSTICAS}

\section{A ) PRUEBAS CUTÁNEAS}

Las pruebas cutáneas tienen excelente aplicación en la identificación de los alérgenos desencadenantes de cuadros asmáticos, rinoconjuntivitis alérgica, dermatitis atópica, urticarias y alergias alimentarias, permitiendo confirmar la hipótesis diagnóstica (2). Considerando que no todas estas enfermedades son de carácter alérgico, las pruebas permiten hacer el diagnóstico diferencial entre cuadros alérgicos y no alérgicos.

También nos permite, al identificar el alérgeno, proponer otros métodos complementarios al diagnóstico de asma, rinitis y conjuntivitis alérgica, como por ejemplo, las pruebas de provocación bronquial, nasal y conjuntival con alérgeno específico, que discutiremos posteriormente.

Las pruebas cutáneas pueden ser clasificadas según el tiempo en que se obtienen los resultados: las de lectura inmediata y las de lectura tardía. Las primeras están relacionadas con el mecanismo de hipersensibilidad inmediata tipo I, mediadas por inmunoglobulina $\mathrm{E}$ (IgE), y las últimas se relacionan con la reacción de hipersensibilidad tardía tipo IV, mediadas por células T, según la clasificación de Geel y Coombs (3).

\section{A.1) PRUEBAS CUTÁNEAS DE LECTURA INMEDIATA}

En este grupo de pruebas están consideradas la de tipo puntura (PT) y la intradérmica (ID). Estas consisten en colocar el extracto alergénico en contacto con las células cutáneas, las que reaccionan liberando mediadores inflamatorios locales que promueven la formación de una pápula con eritema, demostrando la presencia de IgE específica para el alergeno testado.

En la prueba cutánea tipo puntura, son aplicados extractos glicerinados en la epidermis, a través de una punción con una lanceta de $1 \mathrm{~mm}$ de largo, en superficie plana y depilada del cuerpo, preferentemente antebrazo y espalda. El número de extractos a ser utilizados varía según la historia clínica. Es importante conocer la aerobiología local para poder escoger los extractos a ser testados en casos de alergia respiratoria.

Los resultados son obtenidos a los 15 o 30 minutos y la respuesta positiva se manifiesta como una pápula con halo de hiperemia, donde el diámetro de la pápula debe ser $>3 \mathrm{~mm}$, según criterios internacionales de positividad pre-establecidos, independientemente del extracto estandarizado utilizado. Siempre es necesario la utilización de dos controles intrínsecos a las pruebas, siendo el control negativo el diluyente de los extractos y el control positivo una solución de histamina de 10 $\mathrm{mg} / \mathrm{ml}$. Considerando que pueden ocurrir falsos resultados (Tabla $\mathrm{N}^{\circ} 2$ ), la interpretación de los resultados está íntimamente relacionada con la clínica. Se debe evitar el uso de antihistamínicos 72 horas antes de la realización de las pruebas, así como de antidepresivos hasta 7 días antes, pues pueden inducir a resultados falso-negativos.

El método de puntura es considerado más seguro, de menor riesgo de provocar efectos colaterales, pudiendo ser realizado desde temprana edad, según indicación clínica (4). Varios son los métodos empleados para realizar de forma similar la PT, y los estudios demuestran que no hay diferencias estadísticamente significativas en relación al tipo de punctor utilizado (diferentes tipos de lancetas o agujas hipodérmicas) (5). Efectos sistémicos ocurren en $0.03-0.48 \%$ y efectos adversos leves en $0.49 \%$ de los pacientes evaluados. La PT es 
Tabla $\mathrm{N}^{\circ} 2$. Causas de falsos resultados positivos $y$ negativos durante la realización de pruebas cutáneas de lectura inmediata y alergia.

\begin{tabular}{cc}
\hline FALSO POSITIVO & FALSO NEGATIVO \\
\hline $\begin{array}{c}\text { ALTA CONCENTRACIÓN DEL } \\
\text { EXTRACTO }\end{array}$ & EXTRACTO INACTIVADO \\
ERROR DE APLICACIÓN & \\
(ALTA CANTIDAD EN EL ID) & ERROR DE APLICACIÓN \\
(BAJA CANTIDADE Y PROFUNDIDAD & EN EL ID) \\
DERMOGRAFISMO & NO REACTIVIDAD CUTÁNEA \\
CONTAMINACIÓN DEL EXTRACTO & DROGAS ANTIHISTAMINICAS) \\
& MALA INDICACIÓN \\
\hline
\end{tabular}

menos sensible pero más específica que la ID, la cual deberá ser considerada en casos donde la primera sea negativa, pero la historia clínica sea bastante sugestiva para alergia, por motivo de seguridad.

La ID es de menor practicidad clínica, más dolorosa y de mayor riesgo de generar efectos sistémicos. Por presentar alta sensibilidad está sujeta a producir igualmente falsos resultados positivos. En casos de falta de destreza del realizador de la prueba, una aplicación más profunda del extracto induce a resultados falsonegativos. En esta prueba, son utilizados extractos acuosos no glicerinados, y la aplicación se hace directamente en la dermis del antebrazo o de la espalda. La lectura es similar a la prueba de puntura.

En el estudio realizado en una población de pacientes asmáticos en Minas Gerais, Brasil, fueron evaluados varios alérgenos inhalantes ambientales, como: ácaros, hongos, cucaracha y epitelio de animales, observándose que las PT e ID son concordantes en un $89.9 \%$ de los casos (6).

Existe un método alternativo complementario, llamado "prick-by-prick", que utiliza extractos alergénicos brutos o frescos para hacer la búsqueda de alergenicidad. Es muy utilizado en casos de sospecha de alergia alimentaria, tiene alta sensibilidad, pero también está más relacionado a efectos adversos graves, incluyendo choque anafiláctico en pacientes hipersensibilizados. Está indicado cuando el proceso de elaboración del extracto, por ejemplo, frutas y vegetales, promueve la inactivación de proteínas potencialmente alergénicas, lo que conllevaría a falsos negativos en las pruebas cutáneas convencionales. Debe ser considerada la presencia de controles sanos no relacionados, para valorar sus resultados positivos.
Cabe resaltar que a pesar de la seguridad, las reacciones adversas a las pruebas cutáneas son posibles, pudiendo en raros casos llegar a la expresión máxima de la reacción alérgica, el choque anafiláctico; por lo tanto debe ser realizado bajo supervisión médica. Algunos de esos casos están relacionados a la norealización de PT previa a una ID, al "prick-by-prick" y al uso de un número excesivo de alérgenos por prueba (7).

\section{A.2) PRUEBAS CUTÁNEAS DE LECTURA TARDIA TIPO PARCHE}

Las pruebas cutáneas de lectura tardía tipo parche son métodos importantes para identificar agentes que desencadenan cuadros de dermatitis de contacto alérgica o irritativa fundamentalmente, pero también en casos especiales de urticaria por contacto.

Son realizadas en un periodo de tiempo más largo, pues la respuesta alérgica en ese caso es del tipo celular (Linfocitos T) y los resultados se obtienen después de 72 horas del contacto del alérgeno con la piel. Habitualmente son aplicadas en la espalda y el número de extractos a estudiar depende de la historia clínica del paciente. La prueba debe ser aplicada en una área de la piel sin lesiones y con poco vello. En casos especiales, la exposición solar es el desencadenante asociado del cuadro clínico, debiendo el paciente recibir rayos UV en la zona de aplicación de los extractos. Los resultados son referidos según el grado de lesión provocada en la piel, variando del eritema sin vesículas hasta un intenso edema con vesiculación y ulceración en los casos de máxima positividad (8). Una vez identificado el alérgeno o irritante cutáneo, es necesario evitar un contacto futuro para impedir la aparición de nuevas lesiones.

\section{B) PRUEBAS DE PROVOCACIÓN CON ALÉRGENO ESPECÍFICO}

Las pruebas cutáneas presentan un valor predictivo negativo del 80 al $100 \%$, y predictivo positivo de menos de $50 \%$ en los casos de reacción alérgica a medicamentos, haciendo sugerir un diagnóstico que debe ser confirmado con pruebas séricas y pruebas de provocación alérgeno específica.

\section{B.1) PRUEBA DE PROVOCACIÓN ORAL CONTROLADA}

Las pruebas de provocación oral controlada (PPO) son consideradas el "gold standard" del diagnóstico de reacciones alérgicas a alimentos y medicamentos. 
Consisten en la administración de dosis crecientes del producto sospechoso por vía oral y observamos si provoca una reacción alérgica similar a la referida en la historia clínica del paciente. Es un procedimiento diagnóstico definitivo considerándose que demuestra la relación entre manifestación clínica y el producto ingerido (9). Siempre se realiza con el producto sospechoso según la historia clínica y pruebas cutáneas y séricas asociadas. Debe ser realizada en pacientes asintomáticos y está contra-indicada en pacientes con historia clínica de reacción alérgica grave, por el riesgo aumentado de anafilaxia. En la duda, en los casos donde las pruebas cutáneas de lectura inmediata son positivas, asociado a pruebas in vitro séricas positivas (discutidas a continuación), deben ser sometidos a la apreciación de un especialista para determinar la necesidad o no de la realización de la prueba de provocación oral controlada, dependiendo del alérgeno a ser probado. Las PPO son generalmente laboriosas, de larga duración y deben ser realizadas por personal entrenado bajo vigilancia médica, en un local con condiciones para atención de emergencia, debido a que no se puede preveer la severidad de la reacción alérgica que se va a provocar en el paciente durante la prueba. Una PPO no va a determinar el mecanismo inmune involucrado en la reacción presentada por el paciente, pero sí demuestra un mecanismo de "causa y efecto".

\section{B.2) OTROS TIPOS DE PROVOCACIÓN}

Provocación bronquial (PPB) (10), nasal (PPN) y conjuntival (PPC) con alérgeno específico son también métodos auxiliares en el diagnóstico de enfermedades alérgicas, principalmente en casos de asma y rinoconjuntivitis alérgica. En estas pruebas se busca desencadenar la reacción alérgica en el supuesto órgano blanco, utilizándose el alérgeno principal sospechoso. Considerando que son métodos de mayor riesgo en su realización, habitualmente son utilizados en centros académicos dotados de recursos específicos.

\section{C) PRUEBAS SERICAS}

\section{C.1) CUANTIFICACIÓN DE INMUNOGLOBULINA "E" TOTAL}

La cuantificación de IgE total se hace en suero, utilizándose diferentes métodos, enzimáticos o radioactivos. Los individuos de países del Tercer Mundo generalmente están expuestos a los parásitos intestinales (helmintos) que pueden ser estímulos para potenciar la producción policlonal de IgE contra antígenos parasitarios. Aunque la especificidad de estos anticuerpos IgE policlonales no esté determinada, hay evidencias de que no están dirigidos a alérgenos inhalantes (11). La disponibilidad de pruebas in vitro cuantitativos para IgE total y específica asociada a pruebas para la detección de los principales alérgenos inhalantes ambientales permite determinar la asociación entre exposición y sensibilización.

\section{C.2) CUANTIFICACION DE INMUNOGLOBULINA E ESPECÍFICA}

La técnica de determinación de IgE específica fue desarrollada por Wide en 1967, y sigue siendo utilizada como complemento diagnóstico en enfermedades alérgicas mediadas por IgE. La especificidad y sensibilidad varia según el alérgeno evaluado. En algunos casos es útil para determinar el momento adecuado para la reintroducción del alérgeno, después de su exclusión, en los casos de alergia alimentaria. Una prueba sérica negativa no descarta la sensibilización frente a un alérgeno y para dar el diagnóstico final debe ser considerada la historia clínica y pruebas cutáneas específicas asociadas. Existen varios métodos de determinación de IgE específica en suero, como el ELISA (enzyme-linked immunosorbent assay), FAST (fluorescent-allergosorbent test), MAST (múltiple chemiluminescent-allergosorbent test), RAST (radioallergosorbent test). Todos ellos utilizan del principio del "allergosorbent", o sea, alérgeno ligado a un soporte sólido. Si el suero del paciente contiene IgE inmunológicamente específica al alérgeno ligado al soporte sólido, ésta se liga al mismo. Lo que varía en esos sistemas es la forma de reacción que permite revelar el resultado, siendo por ejemplo el ELISA un método enzimático colorimétrico y el RAST un método radioactivo (12). En estudios que realizamos previamente, demostramos que el método de ELISA reverso (rELISA) que utiliza anticuerpo monoclonal alérgeno-específico para medir IgE específica en suero, es más sensible que el ELISA convencional $(70.8 \%)$ y comparable al RAST. Además tiene la ventaja de no utilizar material radioactivo, tornando su realización de menor riesgo y costo (13). Aunque más específicos, esos métodos diagnósticos son menos sensibles cuando son comparados con las pruebas cutáneas, y están indicados precisamente en pacientes con historia de reacción anafiláctica, donde está contra-indicada la realización de la prueba cutánea.

\section{D) OTROS PROCEDIMIENTOS DIAGNÓSTICOS}

Varios otros procedimientos de laboratorio pueden ser utilizados como métodos alternativos de diagnóstico de enfermedades alérgicas, pero son menos específicos o de difícil realización, no presentando relevante aplicación clínica algunos de ellos. En algunos países la cuantificación de óxido nítrico en el gas inhalado ya 
es una realidad, usada en el control de pacientes asmáticos y riníticos. Todavía es una prueba de alto costo operativo, no siendo utilizada de forma rutinaria en países en desarrollo. En nuestro estudio publicado anteriormente (14) describimos una técnica de cuantificación de óxido nítrico en suero, de fácil realización y bajo costo, que puede ser utilizada para ayudar en el diagnóstico y control de tratamiento de individuos asmáticos. Aun así no es un método utilizado de rutina en el control de alergias.

Seguramente ejerce un rol importante en las estadísticas que demuestran el aumento de la prevalencia de alergias en el ámbito mundial, el hecho de que se ha mejorado mucho la capacidad de hacer diagnóstico en las últimas décadas, en forma precoz, eficaz y segura. Las pruebas diagnósticas en alergia son importantes para confirmar el diagnóstico basado en una evidencia clínica, pero cada caso debe ser estudiado de forma individualizada. Aunque no siempre es posible determinar el mecanismo inmunológico implicado en la manifestación clínica del paciente, con las pruebas diagnósticas en alergia es posible por lo menos determinar (o descartar) un mecanismo de "causa y efecto". Estas tienen aplicación clínica importante en enfermedades alérgicas respiratorias y cutáneas, reacciones alérgicas alimentarias y medicamentosas, donde una vez identificado, se podrá evitar el alérgeno desencadenante del cuadro clínico.

Cuando el paciente con historia de reacción sistémica grave presenta pruebas in vitro y pruebas cutáneas negativas que descartan un proceso alérgico, hay que considerar otros diagnósticos, como procesos no inmunológicamente mediados.

Tomando en cuenta las variantes habituales en la realización de estas pruebas, como el personal que las realiza, los métodos y materiales utilizados, variantes ambientales y la sensibilización individual del paciente, se debe considerar la clínica como factor fundamental para analizar y valorar los resultados obtenidos, y concluir el diagnóstico de proceso alérgico.

\section{Correspondencia:}

Dra. Erika Arruda-Chaves

Unidad de alergia e inmunología clínica

Clínica Anglo Americana

Av. Alfredo Salazar Cuadra $3 \mathrm{~s} / \mathrm{n}$

San Isidro - Lima - Perú

arrudachaves@hotmail.com

\section{REFERENCIAS BIBLIOGRAFICAS}

1. Worldwide variation in prevalence of symptoms of asthma, allergic rhinoconjunctivitis, and atopic eczema: ISAAC. The International Study of Asthma and Allergies in Childhood. Steering Commitee. Lancet 1998; 351: 1225-32.

2. Gelber LE, Seltzer LH, Bouzoukis JK, Pollart SM, Chapman MD, Platts-Mills TA. Sensitization and exposure to indoor allergens as risk factors for asthma among patients presenting to hospital. Am. Rev Respir Dis 1993; 147: 573-578.

3. Coca AF, Cooke RA. On the classification of the phenomenon of hypersensitiveness. J Immunol 1923; 8: 163 .En: Imunologia. Roitt I, Brostoff J, Male D. Editora Manole LTDA., Sao Paulo 1997, 22.1-22.17.

4. Dreborg S. Skin testing. Allergy 1993; 48: 473-5.

5. Nelson HS, Rosloniec DM, McCall LI, Ikle D. Comparative performance of five commercial prick skin test devices. J Allergy Clin Immunol 1993; 92(5): 750-6.

6. Moreira MR, Mendonça MR, Gomes Junior E. et al. Sensitization to indoor allergens among asthmatic patients living in Uberlandia, MG. Rev Bras Alerg Imunopatol 2001; 24 (1): 11-21.

7. Novembre E, Bernardini R, Bertini G, Massai G, Vierucci A. Skin-prick-test-induced anaphylaxis. Allergy 1995; 50 (6): 511-3.

8. Allergen skin testing. Board of Directors. American Academy of Allergy and Immunology. J Allergy Clin Immunol 1993; 92(5): 636-7

9. Sampson H. Immunologically mediated food allergy: the importance of food challenge procedures. Ann Allergy 1988; 60: 262-269.

10. Rullo VEV, Rizzo MCV, Solé D, Naspitz CK. Broncoprovocação Específica em Crianças Asmáticas. Rev. Bras. Alergia Imunopatol. 1996; 19 (06): 263-271.

11. Turner KJ, Fedema L, Quinn EH. Non-specific potentiation of IgE by parasitic infections in man. In. Arch Allergy Appl Immunol 1979 ;58:232-236.

12. Rios JBM, Carvalho LP. Alergia alimentar. En: Alergia Clínica: Diagnóstico e Tratamento. Rios JBM, Carvalho LP. Revinter. Rio de Janeiro 1995, 405-423.

13. Silva DA, Gervasio AM, Sopelete MC, et al. A sensitive reverse ELISA for the measurement of specific IgE to Der p 2, a major Dermatophagoides pteronyssinus allergen. Ann Allergy Asthma Immunol 2001; 86 (5): 545-50.

14. Arruda-Chaves E, De Conti D, Tebaldi T. Nitric oxide sera levels as an inflammatory marker in asthma. $J$ Invest Allergol Clin Immunol 2002; 12 (2): 120-123.

Fecha de Recepción : 19-Setiembre-2003

Fecha de Aceptación: 15-Marzo-2004 